\title{
Secured System for the Safety of Two Wheelers
}

\author{
Radeep Krishna .R \\ Senior IEEE member. \\ Electronics and communication engineering \\ Kalasalingam academy of research and education \\ Krishnan kovil,INDIA \\ K.Suresh Babu \\ Electronics and communication engineering \\ Kalasalingam academy of research and education \\ Krishnan kovil,INDIA
}

\begin{abstract}
The proposed model is replication of the deaths caused by accidents. The person who met with accident is lack of remedy at the time of emergency. The major cause for the fatality may be late communication between the victim and the hospitals, and also the lack of human support to the victim at the site of accident. In order to restrict the count of deaths due to accidents, the proposed model "SECURED SYSTEM FOR SAFETY OF TWO WHEELERS" will be useful.
\end{abstract}

The main intention of the proposed system is to reduce the communication barrier between the victim and hospitals. The system is mainly equipped with GSM and GPS for communication, and it is also fortified with alcohol sensor, flex sensor, vibration sensor for making it as a more efficient working system. It is employed with SOS for the safety of women in emergency situations, and also for the fast communication between the victim and nearby security control rooms.

Keywords:- Smart Helmet, GSM, Arduino UNO, Accident Detection, Vibration Sensor, GPS, Women Safety.

\section{INTRODUCTION}

Nowadays the motorcycles are becoming as the part of daily lifecycle. For each and every usage needs the access of the motorcycle from nearest to farthest distances. While the usage is more, the security should also be considered as a major constraint.

The motorcycle should be managed in a way that, both need, and safety should be balanced on each side. The safety issues for the motorcycles are leading to the deaths of the people, who are rider and the pillion rider

The survey made on the bike accidents, concluded that is, the main reason for the accident's occurrence is alcohol consumption and illusion in night lights. The department of safety and health given a census report about accidents of motorcycles, i.e., in a consequent year, nearly 17000 persons are becoming as victims due to bike accidents in a duration of 4 months

\author{
P.V.N.S.Sasidhar \\ Electronics and communication engineering \\ kalasalingam academy of research and education \\ Krishnan kovil,INDIA \\ P.Chakradhar Reddy \\ Electronics and communication engineering \\ Kalasalingam academy of research and education \\ Krishnan kovil,INDIA
}

The proposed system will ensure that the person wore it, made by using the flex sensor fitted inside the helmet. The flex sensor will detect the range and if it exceeds a prefixed range, it will report to the receiver fixed after the plug system of the bike. The readings of flex sensor were constantly sent to the receiver by using a Bluetooth module.

The alcohol sensor will analyse the proportions of alcohol content in the breath of the rider and it will check the level. The values recorded by the alcohol sensor will be continuously sent to the receiver. Based on these values recorded using ultrasonic sensor and the alcohol sensor, the receiver fixed after the plug system controls on and off. The SOS will be activated by the user in the emergency situations for helping from the nearby security control systems.

\section{PROPOSED SYSTEM}

The required components are obtained, and circuits are developed with the required output. All the components should be correctly interfaced with their respective specifications.

\section{System Specifications:}

\begin{tabular}{|c|c|}
\hline Component & Specification \\
\hline Flex sensor & 25 ohms to 1089 ohms \\
\hline Alcohol sensor & MQ-3 \\
\hline Bluetooth module & HC-05 \\
\hline Arduino board & ATMEGA 328 \\
\hline Relay & $5-12 \mathrm{~V}$ \\
\hline GSM module & $800 \mathrm{~A}$ \\
\hline GPS module & SIM28ML \\
\hline Vibration sensor & $10 \mathrm{mV} / \mathrm{g}$ to $100 \mathrm{mV} / \mathrm{g}$ \\
\hline
\end{tabular}

Table 1

\section{$>$ Components Description:}

- Flex sensor:

The Flex sensor is also known to be bend sensor which is useful in deflection measurement. Normally the sensor is employed on the surface of any systems for the sake of measuring bend occurred in it. This sensor will monitor the bending values in the form of change in the resistance. It ranges from $25 \mathrm{ohms}$ to $1089 \mathrm{ohms}$. It is subcategorized into three types namely capacitive flex, velostat 
flex, fibre optic flex. It can also be used as goniometer which also be named as flexible potentiometer.
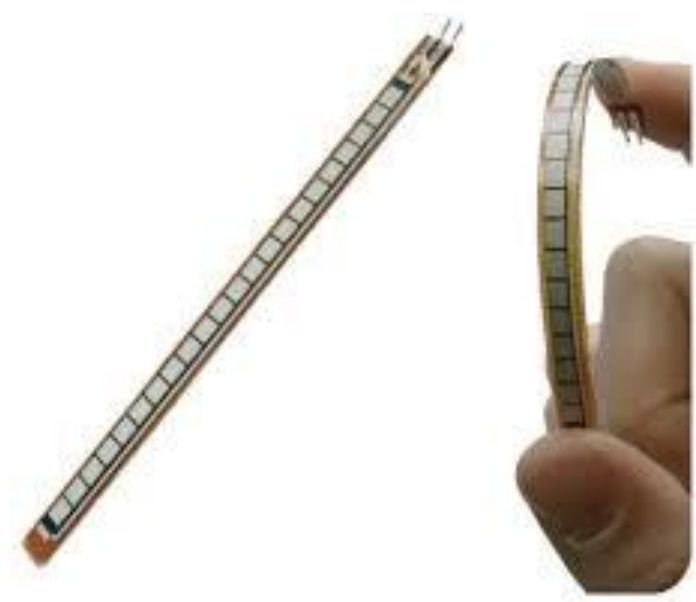

Fig 1:- Estimation of bending.

\section{- Alcohol Sensor:}

The alcohol percentage in particular person is well identified by using the MQ-3 alcohol sensor. The sensor normally works with $5 \mathrm{v}$ supply voltage, due to its less power consuming feature, alcohol sensor is employed in all the major applications.

The sensor can be easily interfaced with all the majorly using micro controllers, like Arduino Uno, raspberry pi etc. It is prefixed to the particular range of values, namely $0.05-10 \mathrm{mg} / \mathrm{L}$. MQ3 is more sensible and less response time. These two features made it more applicable for all the systems.

MQ3 sensor also senses the surrounding temperature and it ranges from -10 to 50 degree centigrade. It is a basic semiconductor type sensor, which is reliable in all situations of working. The alcohol sensor is employed with $\mathrm{SnO} 2$ material for sensing application.

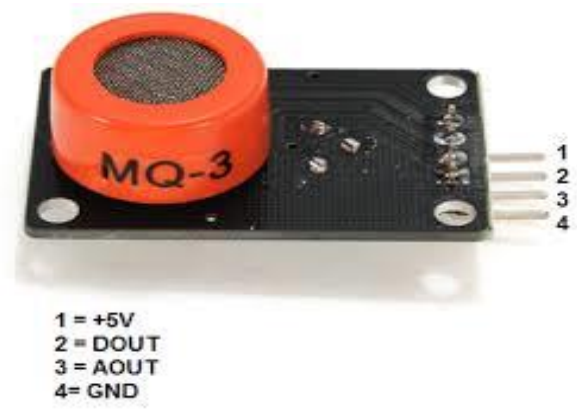

Fig 2:- Alcohol sensor.

\section{- Bluetooth Module:}

The bluetooth module is capable of both transmitting and receiving of data through antennas employed in it. Majorly HC-05 module is utilized for the transmission and reception purpose in this system. The range of frequency it works is lying from 2.4 to $2.485 \mathrm{GhZ}$, and its supply voltage ranges from 3.6 to 6 volts.
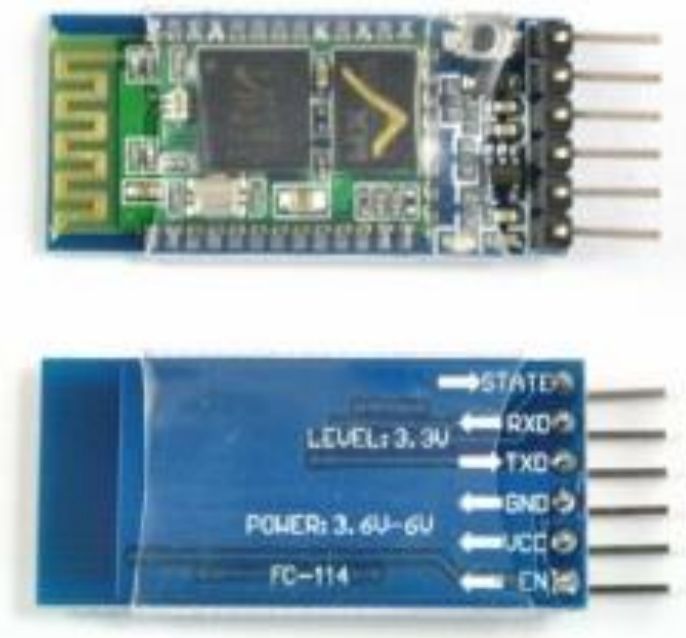

Fig 3:- Bluetooth module.

\section{- Arduino Board:}

Arduino Uno is the board interfaced with the microcontroller beloging to the MEGA family of AVR microcontrollers namely ATMEGA328P. It is the microcontroller board manufactured by ARDUINO Company and programmed by many softwares like arduino IDE software and ATMEL studio. It is mainly a 32 pin Dual Inline Package(DIP) IC used for various applications. The $328 \mathrm{P}$ controller normally employed with bootloader for the external programming. The uno board is having a set of input and output ports which are used for interfacing with various circuits and sensors. It also having 14 digital and 6 Analog pins act as both inputs and output in interfacing with different circuits. The power supply which is very low for the board can be provided by USB cable or external DC power supply. Normally it communicates by using STK500 protocol, and it also differs from many pre-released boards in various aspects like USB serial converter. In all previous boards which are released from Arduino family were using FTDI chip, but here it will be using ATMEGA 16U2 as a USB-to-serial converter, which makes more easy and compatible communication. This Arduino board normally works in clock speed of $16 \mathrm{Mhz}$ and is also comprises of SRAM (Static Random Access Memory) of $2 \mathrm{~KB}$ and EEPROM (electrically erasable and programmable read only memory) of $1 \mathrm{~KB}$ and also the flash memory of $32 \mathrm{~KB}$ in which 500 bytes were taken by bootloader. Always there will be an on-board voltage regulator for controlling the inflow of voltage. The general communication between the systems interfaced with Arduino is done by enabling the digital pins 0 and 1 available on the board, in which the 0 pin is useful for receiving and the pin 1 is useful for transmitting. 


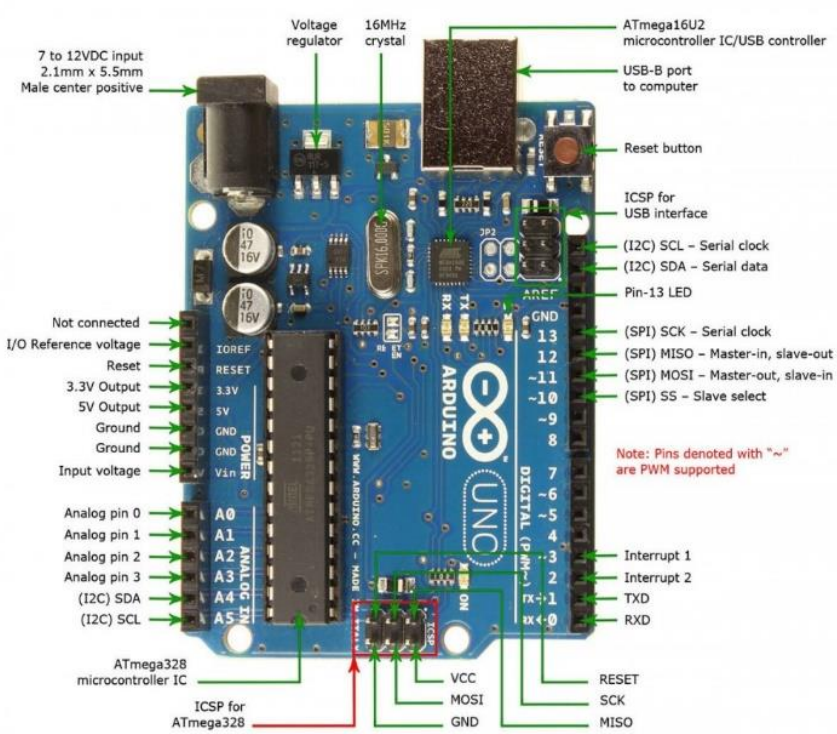

Fig 4:- Arduino UNO.

- Relay:

The switching purpose of electronic systems from one state to another is full filled by using Relay. The various applications are using this relay as a gate device. There are mainly 6 types of relays are available based on the working requirement, namely Hybrid, solid state, magnetic latch, induction, attraction, electro-magnetic.

The electro-magnetic relay is mainly used in the control switches mechanism, these types of relay is applicable to run a low power system.

The relay system belongs to the actuator family, in which electric signal is converted to various forms like, movement, force, sound.

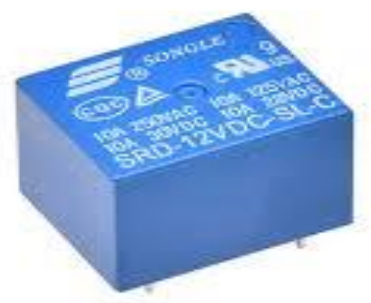

Fig 5:- Relay.

\section{- GPS module:}

It is defined as global positioning system, by which a person can obtain the position information any where in the world. GPS module mainly consists of three segments namely control segment, user segment, space segment. The module which we are using will come under user segment and the antennaused for communication will be considered in the control segment and the satellite used for transmission will be in space segment.
The GPS satellites revolving around the earth at altitude of $21000 \mathrm{kms}$, and the period of working is 12hour intervals. The operating frequency of GPS satellites is 1.2 -1.5 giga hertz. The user segment which replicates the positioning module will generate a C/A code to the space segment.

It is defined as coarse acquisition code for locating. The format of the code generated will be digital binary bits namely 0 's and 1's. the position accuracy depends on GPS satellites around the location of transmitter.

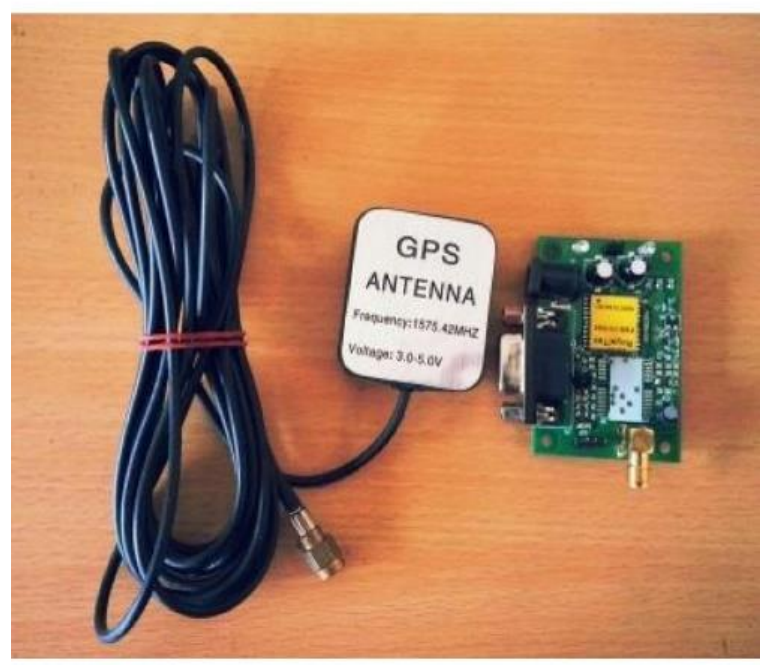

Fig 6:- GPS module.

- GSM module:

GSM is defined as global system for mobile communication. It was introduced at Bell laboratories in the year 1970. It is mostly used in mobile communication system today around the world. It is used for transmitting the mobile voice and net services to the whole world. The operating frequency of GSM is lies between $850 \mathrm{Mhz}$ to $1900 \mathrm{mhz}$.

So, the well-known frequency bands of GSM are $850 \mathrm{MHz}, 900 \mathrm{MHz}, 1800 \mathrm{MHz}, 1900 \mathrm{MHz}$. GSM was developed as an open source of digital system which uses Time Division Multiple Access (TDMA) technique for the purpose of communication. The workflow of Gsm starts with digitizing and reducing the data. Then after it sends through a channel which comprises of two different streams and each stream have its own time slot. The transmitting speed of GSM module is $64 \mathrm{kbps}$ to $120 \mathrm{mbps}$. The GSM system is employed with four types of cells namely Macro, Micro, Pico, and Umbrella. The cell splitting is depended on implementation domain and it also consist of 5 different cell sizes. 


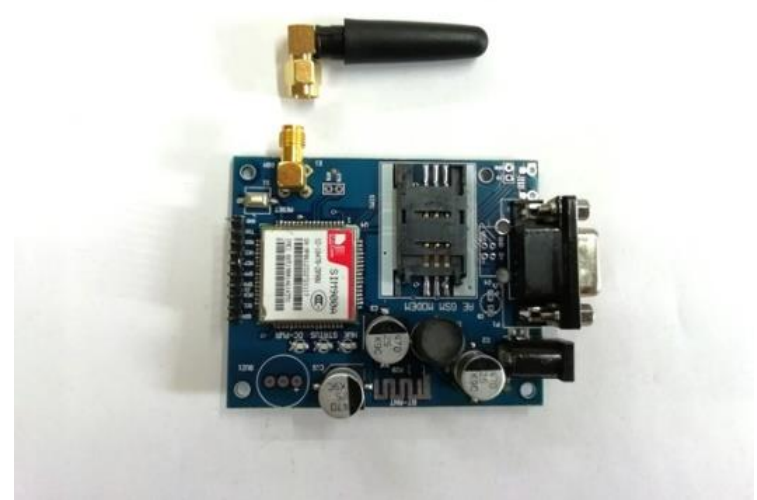

Fig 7:- GSM module

GSM 800A works on the frequencies $850 \mathrm{MHz} 900$ $\mathrm{MHz} 1800 \mathrm{MHz}$ and $1900 \mathrm{MHz}$. It is configurable to AT commands. AT is defined as acceptance test. There are four types of at commands. They are TEST, READ, SET, EXECUTION.

- SOS:

SOS is Morse code which is used internationally the basic version of SOS is developed for the maritime purpose. It attained high profile use in emergencies, so it became as save our souls. It is used to indicate a crisis or conveying the need for action. It is a procedural signal, or it is also termed as prosign. There are particular headers for each type of emergency like loss of life or catastrophic loss of property and other. Other prefixes are used in mechanical breakdown, Medical assistance. SOS signal is also be transmitted in form of flash of light.

\section{- Vibration sensor:}

The vibration sensor is also called a piezoelectric sensor. These sensors are flexible devices which are used for measuring various processes. This sensor uses the piezoelectric effects while measuring the changes within acceleration, pressure, temperature, force otherwise strain by changing to an electrical charge.

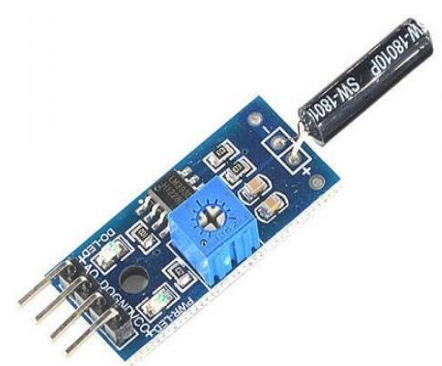

Fig 8:- Vibration sensor.

The sensitivity of these sensors normally ranges from $10 \mathrm{mV} / \mathrm{g}$ to $100 \mathrm{mV} / \mathrm{g}$, and there are lower and higher sensitivities are also accessible. The sensitivity of the sensor can be selected based on the application. So, it is essential to know the levels of vibration amplitude range to which the sensor will be exposed throughout measurements.

\section{SYSTEM INTERFACING}

The connections of all individual components are explained step by step below. The system interfacing is very important for the working of the above specified model.

Step 1:

The Arduino is considered as main important part in the whole system because it is like the operating system for the computer. Now The proposed system is classified into two divisions as Transmitter and Receiver.

\section{Step 2:}

In the transmitter section, the Arduino is connected to different units which are very needed for the working of the system. Mainly the ultrasonic sensor is having 4 pins called $\mathrm{V}_{\mathrm{cc}}$, trig, echo, Gnd. The trig and echo pins are connected to the digital pins assigned in the program. The trig and echo pins are the output and input pins, respectively. The remaining pins $\mathrm{V}_{\mathrm{cc}}$ and Gnd are connected to the respective pins on PCB.

Step 3:

In this system there is two level verification at the level of helmet as flex sensor will detect whether the person wear the helmet or not. The bending of flex sensor will vary the resistance at high level to detect the person wears the helmet or not. The flex sensor will come normal shape when the person removes helmet.

Step 4:

The Rx and Tx pins in Arduino will enables the serial to serial communication. The Bluetooth module (HC-05) is connected to these $\mathrm{Rx}$ and $\mathrm{Tx}$ pins in the Arduino to transmit the bit by bit data. The Bluetooth module hc- 05 is 6 pin structure but 2 pins left unused and the Tx pin of hc05 is connected to Rx pin of Arduino and Rx pin of hc- 05 is connected to Tx of Arduino, which will help in the cross communication. The $\mathrm{Gnd}$ and $\mathrm{V}_{\mathrm{cc}}$ pins are connected to respected pins.

\section{$>$ Step 5:}

In the receiver portion, there is Arduino uno which is connected to the many modules for the successful working of the system. It consists of Arduino uno connected with GPS, GSM modules and also the L293D motor driver connected with the DC motor.

\section{Step 6:}

At the receiver side there is a Bluetooth module which used to receive the data from helmet which is already paired with transmitter using the AT commands. The Rx pin of Bluetooth is connected to Tx pin of Arduino and Tx pin of Bluetooth is connected to Rx pin of Arduino.

\section{Step 7:}

The L293d motor driver is connected to pins assigned in the program with the Arduino. The input pins in the motor driver will drive the motor based on the inputs received from the Arduino pins. The motor will run in 
forward and backward directions based on the inputs. If the person removes the helmet, then the motor stops rotating.

$>$ Step 8:

The GPS module is used to track the location continuously for the safety of the rider. If the rider met with accident or in emergency, then the location will send by the GSM module to the recipients mentioned in the program.

\section{Step 9:}

When the person met with accident the motor of the bike will automatically stops and the accident location is sent to the ambulance and family members.

\section{Step 10:}

The emergency button is kept in the helmet or near the handle. If the person is in emergency, then it is advised to press the button to reach the emergency call centre immediately. It is mainly helpful for the women who are travelling nearby in the town or village using bikes.

\section{IMPLEMENTATION}

The implementation of the system is taken place by deploying the transmitter module in the helmet and the receiver module near the ignition system of the bike. The Arduino at the transmitter part is programmed in a way that whenever the resistance flex sensor is varying and also the ultrasonic sensor also detects lesser distance from the head then it means that the person is wearing the helmet.

The Bluetooth module at the helmet will continuously transmit data to the receiver Bluetooth module as 0's and 1 's. When the person wears the helmet then the distance tracked by ultrasonic sensor will be less than $4 \mathrm{~cm}$, and it transmits 1's to the receiver while the ultrasonic sensor detects distance less than $4 \mathrm{~cm}$.

After receiving the data from the transmitter, receiver will operate on Arduino with received inputs and if it receives 1 then it sends the motor driver to run the motor. If it receives 0 then it sends motor driver to stop the motor. At the same time alcohol sensor detects whether the person consumes alcohol or not. If the person consumes alcohol the bike will stop running.

If the person met with accident, then the vibration sensor will send the acknowledgement to the receiver part that indicates the occurrence of accident. Then with the help of GSM module the Arduino will send the location where accident happened to the recipients whose mobile numbers is provided in the program. Mainly it sends to the ambulance and the nearby blood bank stating the blood group of people.

The SOS button is for the safety of women. When they are in any emergency then they press this button and with the help of GPS module and Arduino the signal is sent to the nearest helpline centre for the help.

\section{RESULT}

As a result, system is implemented and then employed in the helmet for successful completion of the project, on the other hand receiver portion is employed at the ignition system of the bike, which will be useful for the control of bike engine.

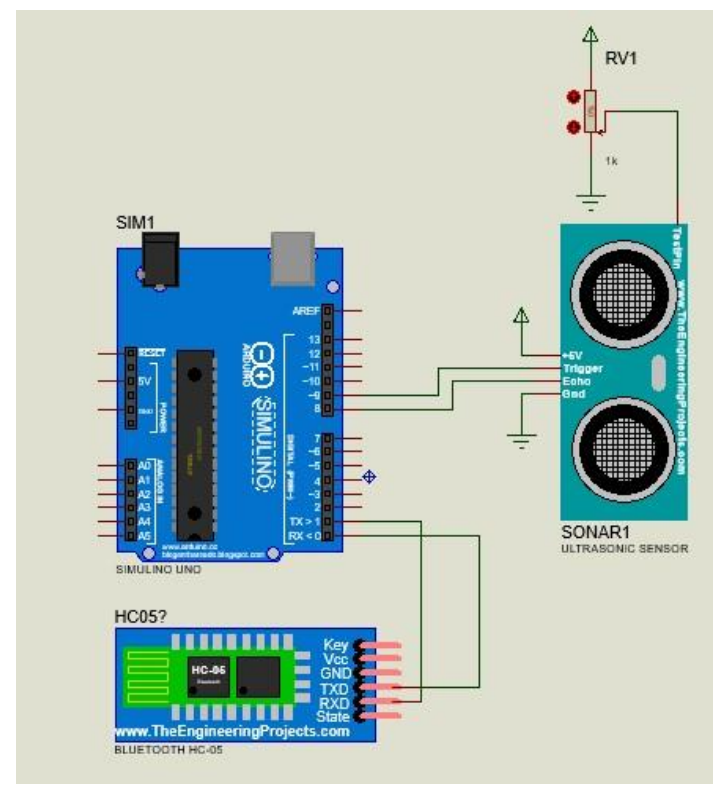

Fig 9:- transmitter simulation image.

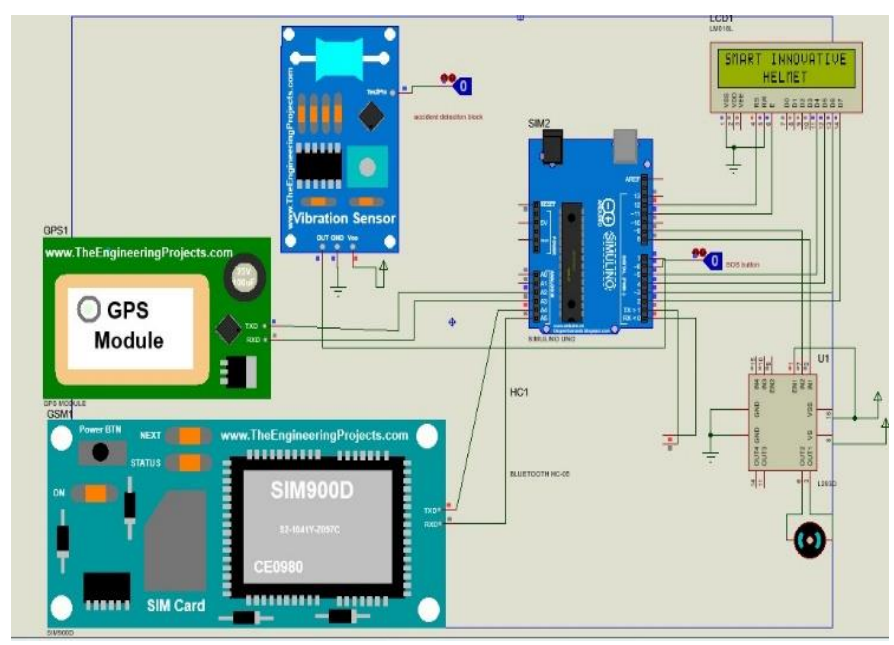

Fig 10:- Receiver simulation image

\section{CONCLUSION}

The effective completion of the system is developed by getting the successful output, for getting that every module of the system will be having a keen role in getting the required throughput. This proposed system includes features like High accuracy, cost effective, giving information about accident within minute, Facilities to call ambulance automatically at the time of accident with greater efficiency. When the person is injured, we must act on time. This model detects the incidence of an accident and makes requirements to sound an alert through the use of a GPS and GSM system. 
The implementation of this system will ensure the person to wear the helmet and also not to be drunk while driving. This will be helping in control of the deaths caused due to not wearing helmet at the site of accident.

\section{FUTURE WORK}

\section{Round Camera:}

The normal camera that is found in the market is not wide enough to record an accident. Accidents could occur from all sides. Hence, if a setup four cameras are placed at 90 degrees to each other forming a cube like structure, which covers all sides.

\section{Attachable Solar Panel:}

Power form solar panel solves all our needs. There would not be any other power source needed for helmet. This would make the helmet eco-friendly.

\section{Bluetooth Earphones:}

Bluetooth earphones helps to feed the navigation to the rider from the mobile phone. Receiving and making emergency calls would be an additional factor to this. With help Government aid, this helmet can be made available for everyone at an affordable rate.

\section{ACKNOWLEDGEMENT}

This project was supported by our department of Electronics and Communication Engineering, Kalasalingam Academy of Research and Education, and we also thank our supporters who provided great expertise during this project. So, the above provided work is true and best of our knowledge attained from the project.

\section{REFERENCES}

[1]. Hartwell, Peter G., and James A. Brug." Smart helmet." U.S. Patent No.6,798,392. 28 Sep.2004.

[2]. Hertz, Cecilia." System for detecting an accident." U.S. Patent No.8,232,881. 31 Jul. 2012.

[3]. Spector, Donald." Combined safety helmet and PA system.” U.S. Patent No. 6,015,160. 18 Jan. 2000.

[4]. Parada, Rita, et al." Helmet-based navigation notifications." U.S. Patent No. 9,146,124. 29 Sep. 2015.

[5]. Kenleigh C. Hobby, Brendan Gowing, P. Matt. 'Smart helmet', US Patent (US 2013/0215281 A1), Aug 22, 2013.

[6]. Mitchell D. Grafinkel, Phil Bart, 'Safety Helmet with Directional and Break Signal Having AM/FM and Two-Way Communication Capability', US patent (6,157,298), Dec 5, 2000.

[7]. Damien Labonde, 'Motor Vehicle Security System', US Patent (5,682,135), Oct. 28, 1997.

[8]. Gary F. Jablonski, Henry J. Ewald, 'Remote Controlled Security System', US Patent $(5,554,977)$, Sep. 10, 1996.

[9]. Ray Fast, 'Global Security System', US Patent (5,497,149), Mar.5, 1996. 